\title{
Impending meningitis in a case of tuberculous otitis media
}

\begin{abstract}
Background: Primary tuberculous otitis is a rare clinical entity and is usually difficult to diagnose. A delay in diagnosis can lead to fatal complications. Mycobacterium tuberculosis is the etiological agent in $0.1 \%$ of the cases of chronic otitis media.

Case presentation: We report case of a child with tuberculous otitis media and cervical spinal involvement. The patient presented with painless purulent ear discharge, hearing loss and signs of meningeal irritation. The biopsy of specimen taken during the mastoidectomy procedure led to the diagnosis.
\end{abstract}

Conclusion: Tuberculous otitis media is an indolent disease and can cause significant damage to middle ear and surrounding structures if left untreated. It is a diagnostic challenge and clinicians should be aware of similar association as it may result in delayed treatment with fatal consequences.
Volume 8 Issue I - 2017

\section{Anjali Venugopal, Akanksha A Saberwal, Haritosh K Velankar,Yogesh G Dabholkar, Yessukrishna Shetty}

Department of ENT DY Patil Hospital and Research Centre, India

Correspondence: Anjali Venugopal Department of ENT DY Patil Hospital and Research Centre 70I A wing Apsara Building Plot No 5 I Sector 17 Vashi Navi Mumbai 400703 Maharashtra, India, Tel+9|-982 I483007, Email dravk@ymail.com

Received: March 20, 2017| Published: July 13, 2017

Keywords: tuberculous otitis media, meningeal irritation, hearing loss

\section{Introduction}

Primary tuberculous otitis media is a rare disease and it results in silent mastoiditis. It is an atypical cause of chronic suppurative infection of middle ear and constitutes for $0.05 \%$ to $0.9 \%$ of chronic otitis media. ${ }^{1}$ It presents as painless otorrhea that fails to respond to the usual antibiotic treatment. It is also associated with hearing loss that is disproportionate to the extent of the disease. ${ }^{2}$

The diagnosis is intricate when tubercular otitis co-exists with bone or central nervous system involvement. It is also notable that whenever tubercular otitis occurs, it is associated with complications like early destruction of the middle ear conductive mechanism, facial paralysis, cochlear involvement with labrynthitis, bone destruction with thrombosis of veins and intracranial infection like meningitis. ${ }^{3}$ When the infection occurs in bones, thoraco-lumbar vertebrae is more commonly involved than cervical vertebrae. ${ }^{4}$ The diagnosis is based on histo-pathological examination.

A high level of clinical suspicion is required for early diagnosis and prompt anti-tubercular treatment should be started to prevent possible complications.

\section{Case report}

A six-year-old male came to our institution with complaints of neck stiffness with pain and headache for the past 15 days. Patient had a history of left ear discharge associated with decreased hearing for a year. The character of discharge was painless, scanty, continuous, purulent and foul smelling which did not significantly resolve with local or systemic antibiotics. It was associated with neck pain that was restricting neck movements. There was no significant past or family history. Clinical examination showed afebrile patient with neck rigidity. Brudzinski's and Kernig's sign were positive. Otoscopic examination revealed a postero-superior retraction pocket of the left tympanic membrane. Ear swab for culture and sensitivity showed no bacterial growth. Audiometric tests revealed moderate conductive hearing loss in left ear. Schuller's view X-ray mastoid showed loss of pneumatization of left mastoid region. Routine blood investigations and chest $\mathrm{x}$-ray were unremarkable.
Patient was admitted and started on empirical intravenous antibiotics. Mantoux test was negative. An attempt of lumbar puncture was unsuccessful. High Resolution Computed Tomography of temporal bone was suggestive of an ill defines iso-dense heterogeneously enhancing lytic expansile lesion involving left jugular bulb causing erosion of squamous part of temporal bone, clivus, mastoid part of temporal bone with thrombosis of left sigmoid sinus and internal jugular vein (Figure 1). Post contrast Magnetic Resonance Imaging of brain revealed an ill-defined moderately heterogeneous enhancing lesion of left jugular bulb causing erosion of squamous part of occipital bone and extending into pre and para vertebral space up to level of $\mathrm{C} 1$ vertebra and causing destruction of mastoid part of temporal bone with thrombosis of left sigmoid sinus and internal jugular vein (Figure 2). Cranial Magnetic Resonance Imaging venography showed thrombosis in left sigmoid sinus and internal jugular vein (Figure 3). Blood investigations ruled out the presence of hypercoagulable state in our patient.

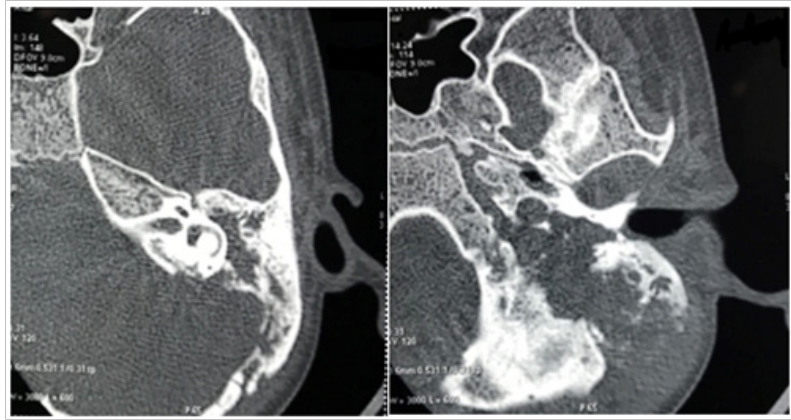

Figure I High Resolution Computed Tomography of temporal bone: Erosion of squamous part of temporal bone, clivus and mastoid part of temporal bone on left side.

Patient was posted for immediate surgery under antibiotic cover. A left modified radical mastoidectomy was done. Peri-operatively granulations were seen in the middle ear along with soft tissue growth seen in external auditory canal and hypotympanum extending to mastoid cavity and eustachian tube. Infected left sigmoid 
sinus and internal jugular vein thrombus was evacuated (Figure $4 a, 4 b, 4 c)$. Intra-operative swabs collected for Acid fast bacilli, culture and sensitivity and Zeil-Nelson staining reported negative. Histopathology report of biopsy of middle ear granulations revealed multifocal epitheloid granulomas, multinucleate histiocytic giant cells and abundant lymphoplasma cell infiltrate with caseous necrosis (Figure 5). Postoperatively, patient was immediately started on antitubercular therapy in addition to adjunct treatment with cervical collar stabilization. Within two weeks of starting antitubercular therapy, patient showed remarkable recovery with disappearance of otologic complaints and relief of neck pain. Patient maintained regular 3-month follow-up which showed well epithelialized cavity with no recurrence.

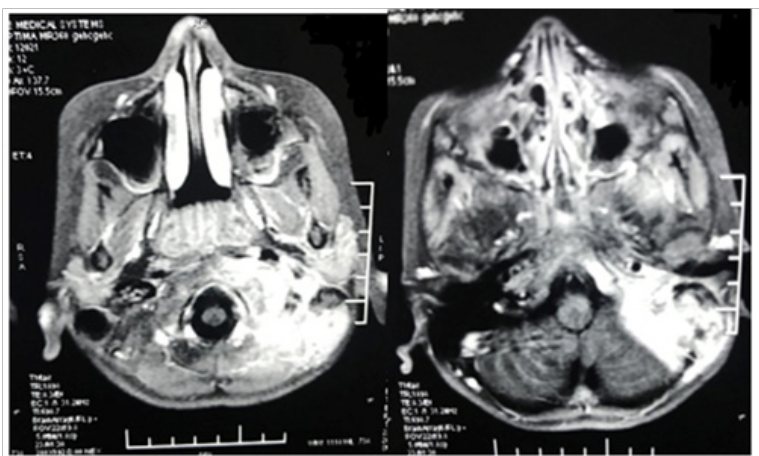

Figure 2 Post contrast Magnetic Resonance Imaging of brain: Destruction of mastoid part of temporal bone with erosion of squamous part of temporal bone and thrombosis of left sigmoid sinus and left internal jugular vein.

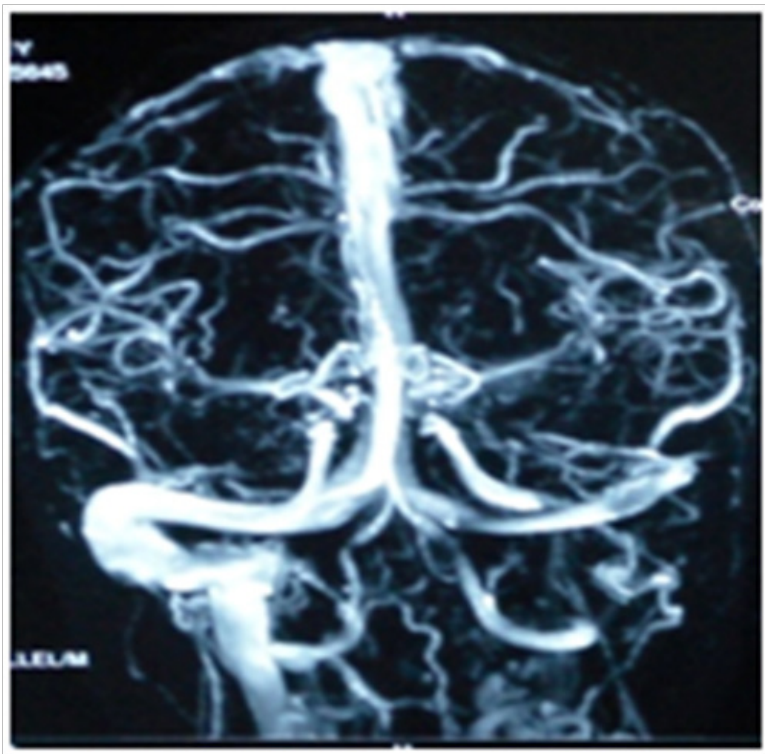

Figure 3 Cranial Magnetic Resonance Imaging Venography:Absent flow in left side.

\section{Discussion}

Tuberculous otitis media in adults most commonly occurs in association with advanced pulmonary or extra-pulmonary tuberculosis but in children it may occur in isolation. ${ }^{5}$ At the turn of century, $3-5 \%$ of all chronic suppurative otitis media cases had tuberculosis and this incidence reduced drastically after the introduction of Anti-tubercular therapy. ${ }^{6} 2.8 \%$ of all cases of suppurative otitis media were due to tuberculosis according to a study conducted by Turner and Eraser in $1915^{7}$. Study by Winer GM et al., ${ }^{8}$ has suggested an incidence rate of around $0.04 \%$. Latest incidence reports a $0.37 \%$ incidence of tubercular otitis amongst all pulmonary and extra-pulmonary tuberculosis. ${ }^{5}$

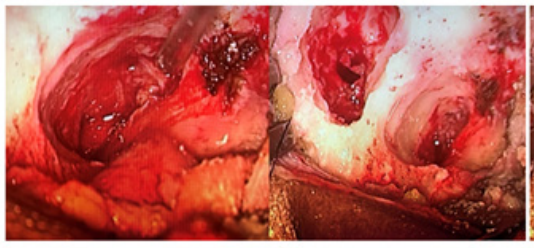

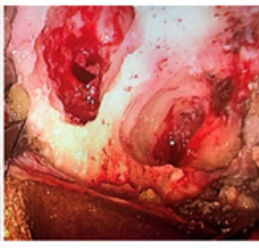

Figure 4a-4c Intra-operative pictures of granulations seen during left modified radical mastoidectomy procedure.

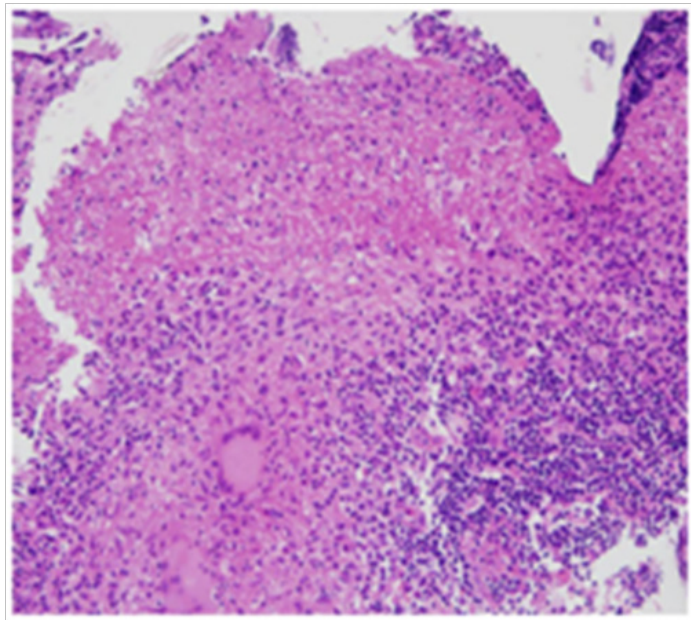

Figure 5 Microscopy of biopsy from left middle ear granulations: Epitheloid granulomas, multinucleate histiocytic giant cells with caseous necrosis.

It is stated that the most common mode of spread of tuberculosis to the central nervous system is by haematogenous route. This focus of infection may be either in the primary site of the lungs or infected middle ear. A potential route of infection is due to direct spread from middle ear through the anatomical barriers through tegmen tympani where it may directly invade the dura mater and middle cranial fossa. Other mode of spread is through the mastoid and then invading sigmoid sinus. The close proximity of these structures aids in rapid spread. ${ }^{9}$

The clinical onset of tuberculous otitis media is insidious and variable. Prompt diagnosis is not easy because of its low prevalence, equivocal clinical signs and false negative culture. Although it may present with a wide range of symptoms, the main symptom is painless ear discharge not responding to the conventional antibiotic therapy. The pattern of hearing loss is inordinate with extent of the disease and conductive hearing loss is commonly present. A characteristic clinical finding is granulomatous middle ear mucosa which is pale and a vascular. Multiple perforations of the tympanic membrane cannot be substantiated as a hallmark of tuberculous otitis media. ${ }^{10}$ As the pathologic process advances inexorably, the granulations lead to attico-antral blockage and destruction of nearby tissues such as skull base or the tegmen tympani, leading to tuberculous meningitis or skull base osteomyelitis. ${ }^{10,11}$

A comprehensive diagnostic workup must include radiography of chest and mastoid, smears and cultures of aural discharge, skin test, blood investigations and audiogram. Imaging studies with clinical and other complementary tests, can strengthen the suspected diagnosis. 
Radiology of mastoid bone may not be of significant help due to nonspecific increased soft tissue density. High resolution computerized tomography of temporal bone proves to be the best imaging modality and may reveal a soft tissue density in middle ear with clouding of mastoid air cells, erosion of mastoid or petrous part, destruction of ossicles or involvement of auditory nerve. Sclerosis of mastoid is denser and more widespread in tuberculous otitis media. ${ }^{12}$ Munoz et al., ${ }^{13}$ advocated the role of Magnetic resonance Imaging in diagnosing tuberculous otitis media and stated that low T2 signal with cerebroid like appearance and extensive coalescent material that enhances on post contrast studies is particularly not seen in usual forms of chronic otitis media. Magnetic Resonance Imaging of Brain with contrast provides better soft tissue definition and sensitivity to blood flow rates compared to Computed Tomography scanning. It can identify low density intraluminal thrombus because of contrast uptake, soft tissue swelling around the sinus and veins and a distended vein proximal to thrombus. ${ }^{13}$ Also, fragmentation or destruction of vertebral body and involvement of clivus and occipital condyle is observed. Histologic studies of tuberculous lesions generally reveal caseous foci which are surrounded by histiocytic or epitheloid cells, multinucleated inflammatory cells, and mixed acute and chronic inflammatory cells along with fibrous granulation tissue. A definitive diagnosis requires identification of the acid-fast bacilli by either culture or tissue staining. ${ }^{14}$

Anti-tuberculous drugs for a period ranging from 6 to 12 months are the mainstay for treatment of tuberculous otitis media. ${ }^{4}$ The therapeutic regimen for our patient was in accordance with the treatment guidelines for Tuberculosis in India. The current standard therapy (Category II) includes a 5 drug regimen in the first two months (Isoniazid, Rifampicin, Pyrazinamide, Ethambutol and Streptomycin) followed by 4 drug regimen in the next one month (Isoniazid, Rifampicin, Pyrazinamide and Ethambutol) and 3 drug regimen for the later five months (Isoniazid, Rifampicin and Ethambutol). ${ }^{15}$ In the presence of sinus or jugular vein thrombosis, hypercoagulable state which may be either inherited or acquired, must be investigated to start anticoagulation therapy along with Anti-tubercular treatment. ${ }^{16}$ Surgery is indicated for complicated cases such as those involving subperiosteal abscess of mastoid, postauricular fistulas, facial palsy or central nervous system extension of infection. ${ }^{1,8}$ When surgery is combined with adequate chemotherapy, there are chances of healing with good prognosis. In our patient, anti- tubercular treatment with supportive treatment from the orthopaedic surgeon and physiotherapist proved adequate.

\section{Conclusion}

Tuberculous otitis media is an unusual disease and should not be neglected. A high index of clinical suspicion is required. The use of available diagnostic techniques will favour rapid and accurate identification of specific pathology. Specific therapy should be initiated as early as possible to prevent further complications and dissemination of infection. Early diagnosis would no doubt reduce the attendant morbidity and mortality associated with the disease before it runs its full course.

\section{Acknowledgements}

We would like to acknowledge the Doctors and Nursing staff in our department who participated in the treatment of this patient.

\section{Conflicts of interest}

All authors declare to have no conflict of interest.

\section{Funding}

None.

\section{References}

1. Grewal DS, Baser B, Shahani R N, et al. Tuberculous otitis media presenting as complications: Report of 18 cases. Auris Nasus larynx. 1991;18(3):199-208.

2. Yaniv E. Tuberculous otitis media: A clinical record. Laryngoscope. 1987;97(11):1303-1306.

3. Windle-Taylor PC, Bailey CM. Tuberculosis otitis media: A series of 22 patients. Laryngoscope. 1980;90(6 Pt 1):1039-1044.

4. Ikem IC, Bamgboye EA, Olasinde AA. Spinal Tuberculosis: A 15-year review at OAUTHC, lle-Ife. Niger Postgraduate Med J. $2001 ; 8(1): 22-25$.

5. Michael J Gleeson, Ray C Clarke. Scott Brown's Otorhinolaryngology and Head and Neck surgery (7th edn). Edward Arnold Publishers Ltd., London, UK. 2008. p.3900.

6. Aase M, Braee-Pedersen C. Primary Tuberculos Otitis Media. J laryngol Otol. 1971;85(11):1195-1200.

7. Turner A L, Eraser J S. Tuberculsis of the middle ear cleft in children. $J$ Laryngol Otol . 1915:30(1):209.

8. Weiner G M, O'Connell J E, Pahor A L. The role of surgery in tuberculous mastoiditis: Appropriate chemotherapy is not always enough. J Laryngol Otol. 1977;111(8):752-753.

9. Tandon PN, Ramamurthi R. Ramamurthi and Tandon's Textbook of Neurosurgery (3rd edn), Jaypee Brothers Medical Publishers Ltd., New Delhi, India. 2012. p.725-752.

10. Plester D, Pusalkar, Steinbach E. Clinical Records, Middle ear tuberculosis. J laryngol Otol . 1980;94 (12):1415-1421.

11. Awan MS, Salahuddin I. Tuberculous otitis media: two case reports and literature review. Ear Nose and Throat J. 2002;81(11):792-794.

12. Chirch L M, Ahmad K, Spinner W, et al. Tuberculous otitis media: Report of 2 cases on Long Island, N.Y., and a review of all cases reported in the United States from 1990 through 2003. Ear Nose Throat J. 2005; 84(8): 488-490.

13. Munoz A, Ruiz-Contreras J, Jiemenez A, et al. Bilateral tuberculous otomastoiditis in an immunocompetent 5-years-old child: CT and MRI findings. Eur Radiol. 2009;19(6):1560-1563.

14. Cleary KR, Batsakis JG. Mycobacterial disease of the Head and Neck: Current perspective. Ann Otol Rhinol Laryngol. 1995; 104(10 Pt 1):830-833.

15. National Guidelines on Diagnosis and Treatment of Pediatric Tuberculosis. 2012.

16. Zangari P, Messia V, Viccaro M, et al. Genetic prothrombotic factors in children with otogenic lateral sinus thrombosis: five case reports. Blood Coagul Fibrinolysis. 2012;23(2):158-163. 